\title{
p53, miR-34a and EMP1-Newly Identified Targets of TFF3 Signaling in Y79 Retinoblastoma Cells
}

\author{
Maike Busch ${ }^{1, *(D)}$, Stefan Klein ${ }^{1}$, Jan Große-Kreul ${ }^{1}$, Oliver Scheiner ${ }^{1}$, Klaus Metz ${ }^{2}$, \\ Harald Stephan ${ }^{3}$ and Nicole Dünker ${ }^{1}$ (D) \\ 1 Institute of Anatomy II, Department of Neuroanatomy, Medical Faculty, University of Duisburg-Essen, \\ 45122 Essen, Germany \\ 2 Institute of Pathology, Medical Faculty, University of Duisburg-Essen, 45122 Essen, Germany \\ 3 Division of Haematology and Oncology, Children's Hospital, University of Duisburg-Essen, \\ 45122 Essen, Germany \\ * Correspondence: maike.busch@uk-essen.de; Fax: +49-(0)201-723-5635
}

Received: 2 August 2019; Accepted: 21 August 2019; Published: 24 August 2019

\begin{abstract}
Trefoil factor family peptide 3 (TFF3) is supposed to have tumor suppressive functions in retinoblastoma (RB), but the functional pathway is not completely understood. In the study presented, we investigated the downstream pathway of TFF3 signaling in Y79 RB cells. Results from pG13-luciferase reporter assays and western blot analyses indicate induced p53 activity with an upregulation of miR-34a after TFF3 overexpression. Expression levels of the predicted miR-34a target epithelial membrane protein 1 (EMP1) are reduced after TFF3 overexpression. As revealed by WST-1 assay, BrdU, and DAPI cell counts viability and proliferation of Y79 cells significantly decrease following EMP1 knockdown, while apoptosis levels significantly increase. Opposite effects on Y79 cells' growth could be shown after EMP1 overexpression. Caspase assays showed that EMP1 induced apoptosis after overexpression is at least partially caspase-3/7 dependent. Colony formation and soft agarose assays, testing for anchorage independent growth, revealed that EMP1 overexpressing Y79 cells have a significantly higher ability to form colonies. In in ovo chicken chorioallantoic membrane (CAM) assays inoculated EMP1 overexpressing Y79 cells form significantly larger CAM tumors. Moreover, miR-34a overexpression increases sensitivity of Y79 cells towards RB chemotherapeutics, however, without involvement of EMP1. In summary, the TFF3 signaling pathway in Y79 RB cells involves the activation of p53 with downstream induction of miR-34a and subsequent inhibition of EMP1.
\end{abstract}

Keywords: TFF3; EMP1; p53; miR34a; retinoblastoma; chemotherapy; tumor formation; CAM assay

\section{Introduction}

The retinal tumor retinoblastoma (RB) is the most common primary intraocular tumor in childhood with an incidence of 1:16,000 livebirth [1]. Retinoblastoma arises in the developing retina as a part of the central nervous system (CNS) [2]. The molecular mechanisms underlying RB development have been intensively studied during the last decades and it has been postulated to be a multi-step process of progression from normal retinal tissue towards RB cells [3]. Retinal cells homozygous for RB1 loss can form benign tumors called retinomas, but further genomic changes are needed to form retinoblastoma [4]. Beside several potential target genes, MYCN amplifications [5] and dysregulated miRNAs [6] play a role in RB development. Untreated RB will grow and extend beyond the eye and undergo metastatic spread, commonly into regional lymph nodes, bone and CNS [2].

Treatment of RB ranges from enucleation of the affected eye to eye salvage strategies including focal therapy (laser, cryotherapy), brachytherapy and/or chemotherapy. Chemotherapy can be applied 
systemically, commonly using an intra-arterial [7] and/or intravitreal VEC (vincristine, etoposide, and carboplatin) injection of chemotherapeutics [8]. Chemotherapeutic treatment of RB is, however, limited not only by drug-related side effects, but also by developing drug resistances. Therefore, developing strategies to improve RB therapies for instance by sensitizing RB cells towards chemotherapeutics is a major challenge.

Recent studies by our group suggest that trefoil factor family (TFF) peptides might be potential new candidates for supplementary therapeutic options for retinoblastoma along with common chemotherapy. Three TFF peptides, TFF1, TFF2, and TFF3, have been identified in mammals so far (reviewed in refs. [9-14]). They are characterized by a clover leaf-like disulfide structure, the so-called TFF domain. TFFs are normally expressed in the gastrointestinal tract with functions in protection and maintenance of epithelial surfaces [10]. They are, however, also expressed in the central nervous system, in human ocular tissues and in the murine retina (for review see [13]). Previous studies by our group revealed that only TFF3, but not TFF1 is expressed in the healthy human retina [15]. Additionally, TFF peptides are either overexpressed or missing in different human tumor entities and tumor cell lines, e.g., gastric, breast, and ovarian cancer and retinoblastoma cells [13]. Furthermore, TFF3 expression correlates with the tumor grade in hepatocellular carcinoma [16] and is a marker for poor prognosis in gastric carcinoma [17]. We could demonstrate that the expression of TFF3 in retinoblastoma cell lines is regulated epigenetically [18] and that forced TFF3 expression leads to reduced RB tumor growth, viability and tumorigenicity as well as enhanced caspase-dependent apoptosis induction in human RB cell lines [19].

However, the downstream targets of TFF3 signaling during RB development and progression have not been investigated so far. Soutto et al. [20] showed that TFF1 activates p53 in gastric epithelial cell lines. Our group confirmed the activation of p53 in TFF1 overexpressing RB cell lines [21], but it still remained to be determine whether this signaling cascade also applies for TFF3. MicroRNA 34a (miR-34a) has been found to be a direct transcriptional target of p53 with canonical p53 binding sites in its promotor region [22]. Moreover, in RB cells overexpression of miR-34a leads to enhanced chemosensitivity against the common RB chemotherapeutics vincristine, etoposide and carboplatin [23]. Moreover, the integral membrane glycoprotein epithelial membrane protein 1 (EMP1) is a predicted target gene of miR-34a. EMP1 has been shown to be regulated by miR-34a in RB cell lines [24] and is supposed to be a potential biomarker for tumor diagnosis and prognosis of several cancers [25]. Thus, we hypothesized that EMP1 might be a downstream target of TFF3 mediating its anti-proliferative and pro-apoptotic effects in RB cells.

In the study presented, we set out to prove that p53, miR-34a and epithelial membrane protein 1 (EMP1) are downstream targets of TFF3 signaling in the retinoblastoma cell line Y79.

\section{Results}

\subsection{TFF3 Overexpression Activates $p 53$ and miR-34a and Inhibits EMP1 Expression}

We performed a lentiviral overexpression of TFF3 in Y79 RB cells (Figure 1A) and analyzed different potential target genes involved in TFF3 signaling to investigate the underlying mechanisms of TFF3's tumor-suppressive functions. We first analyzed the transcriptional activation of p53 by luciferase reporter assay. For this purpose, Y79 cells were transiently transfected with pG13-Luc (with p53 binding site) and the non-responsive reporter pG15- Luc (with a mutant p53 binding site) along with TFF3 or an empty vector control. Compared to control cells the relative luciferase-activity of the p53 responsive reporter was significantly increased in TFF3 overexpressing cells (Figure 1B). western blot analysis revealed a 2-fold increase in p53 expression in TFF3 overexpressing Y79 cells (Figure 1C). 

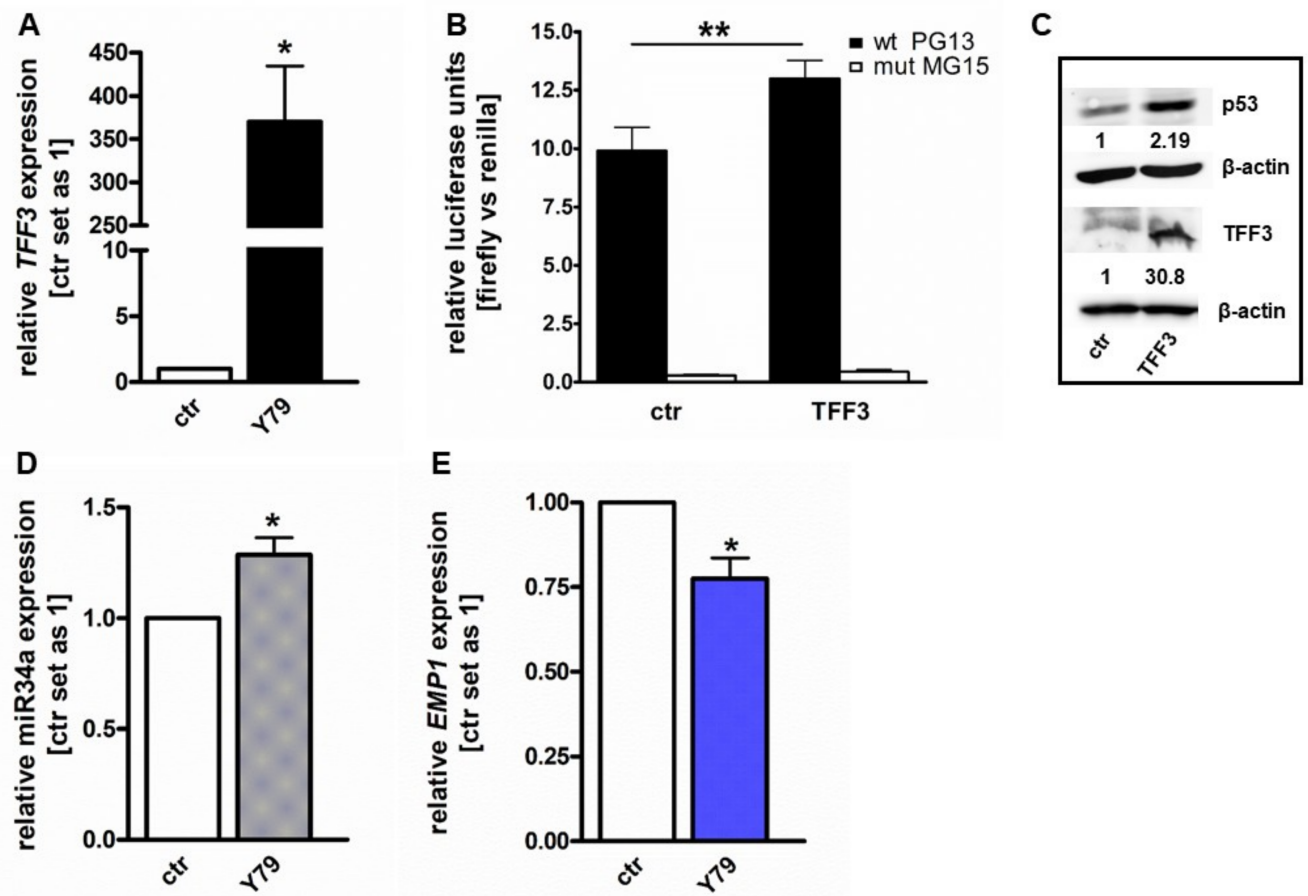

Figure 1. TFF3 overexpression enhances p53 transcriptional activity and protein expression and increases miR-34a expression with downstream reduction of EMP1 in Y79 RB cells. (A) Quantitative Real-time PCR confirmation of TFF3 lentiviral overexpression (Trefoil factor family peptide 3 (TFF3)) in Y79 cells compared to control cells (ctr). (B) Luciferase assays were performed with Y79 cells transiently transfected with TFF3 or empty vector control (ctr) in addition to wild-type PG13-Luc (wt PG13) or mutant MG15-Luc (mut MG15). Forced TFF3 expression leads to an increased luciferase signal upon p53 promotor activation in Y79 cells. (C) Western blot analysis showing increased p53 and TFF3 protein levels after TFF3 overexpression (TFF3). The indicated intensity ratios of p53 and TFF3 protein levels relative to $\beta$-actin levels were calculated using ImageJ software. (D,E) Quantitative real-time PCR analysis of miR-34a and EMP1 expression levels in Y79 cells compared to control cells after lentiviral TFF3 overexpression (ctr). Values are means of at least 3 independent experiments \pm SEM. ${ }^{*} p$-value $<0.05,{ }^{* *} p$-value $<0.01$; statistical differences compared to the control group calculated by Student's $t$-test or one-way ANOVA and Newman-Keuls post test.

Next, we analyzed the expression of miR-34a after TFF3 overexpression. Quantitative Real-time PCR analysis revealed significantly increased levels of miR-34a expression after TFF3 overexpression in Y79 cells (Figure 1D). Moreover, Real-time PCR analyses of Y79 RB cells revealed significantly reduced EMP1 expression levels following TFF3 overexpression in comparison to control cells (Figure 1E). All RB cell lines except for Rbl13 and all primary patients' tumor samples analyzed exhibit higher endogenous miR34a expression levels and lower EMP1 expression levels compared to a healthy human retina pool (Figure S1).

\subsection{EMP1 Knockdown Inhibits Growth and Induces Apoptosis in Y79 Cells}

A previous study by our group demonstrated that TFF3 overexpression reduces viability and proliferation and enhances apoptosis in human RB cell lines [19]. Here we demonstrate that EMP1 levels are downregulated after TFF3 overexpression (Figure 1E). Hypothesizing that EMP1 triggers the effects seen after TFF3 overexpression, we knocked EMP1 down in order to prove that reduced EMP1 levels provoke the same effects as TFF3 overexpression. EMP1 knockdown was confirmed by Real-time PCR (Supplementary Figure S2A) and western blot analysis (Figure 2A). Y79 cells with reduced EMP1 expression levels exhibited significantly lower cell viability (Figure 2B) and displayed 
significantly decreased proliferation levels as revealed by BrdU cell counts (Figure 2C). Moreover, after EMP1 knockdown a significant increase in apoptosis levels was detectable (Figure 2D).

A

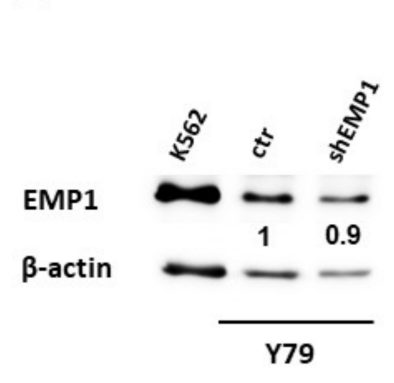

B

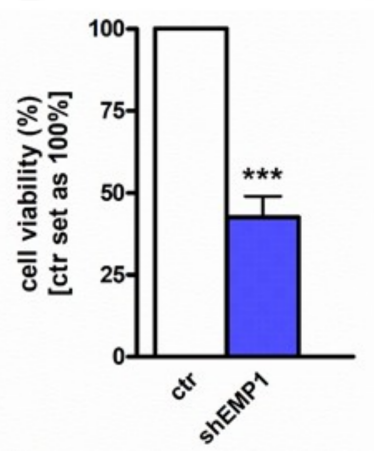

C

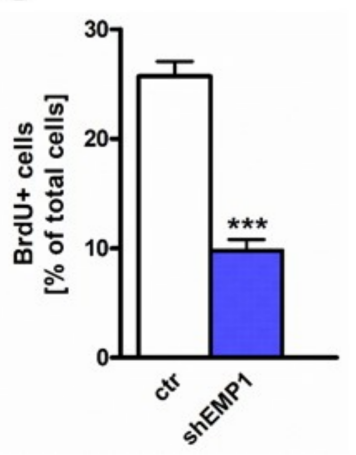

D

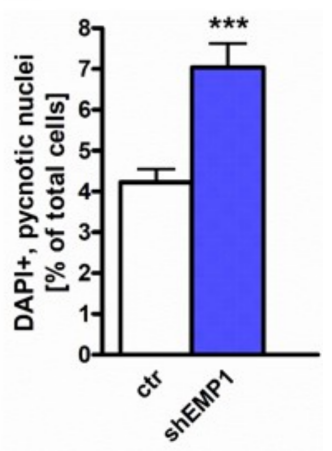

Figure 2. Epithelial membrane protein 1 (EMP1) knockdown leads to reduced cell viability and proliferation and induces apoptosis in Y79 RB cells. (A) Western blot data confirmed decreased EMP1 protein levels after EMP1 knockdown (shEMP1) in Y79 cells. The CML cell line K562 served as an EMP1 positive control, B-actin as a loading control. (B,C) Stably EMP1 knockdown Y79 RB cells (shEMP1) showed significantly decreased viability and proliferation levels compared to control cells (ctr) as revealed by (B) WST-1 assays and (C) BrdU stains. (D) EMP1 knockdown Y79 cells (shEMP1) displayed a significantly increased apoptosis rate compared to control cells (ctr) as revealed by DAPI stains. Values are means of 3 independent experiments \pm SEM. ${ }^{* * *} p$-value $<0.001$ statistical differences compared to the control group calculated by Student's $t$-test.

\subsection{EMP1 Overexpression Induces Growth and Inhibits Apoptosis in Y79 Cells}

As EMP1 knockdown results in a strong anti-proliferative effect, hampering any further detailed in vitro and subsequent in vivo analyses, we analyzed the effects of EMP1 overexpression in Y79 cells, expecting to reveal the opposite effects.

EMP1 overexpression was confirmed by Real-time PCR (Supplementary Figure S2B) and western blot analysis (Figure 3A). EMP1 overexpressing Y79 cells exhibited a significantly higher cell viability (Figure 3B) and displayed significantly increased growth as revealed by BrdU cell counts (Figure 3C) as well as growth curve analyses (Figure 3D). Moreover, after EMP1 overexpression a significant decrease in apoptosis levels was detectable (Figure 3E), which turned out to be caspase3/7-dependent (Figure 3E). 
A

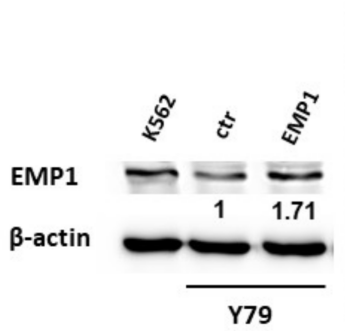

Y79
B

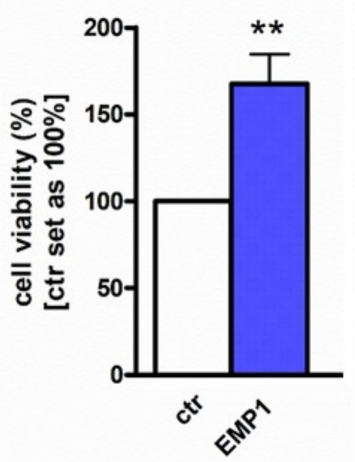

C
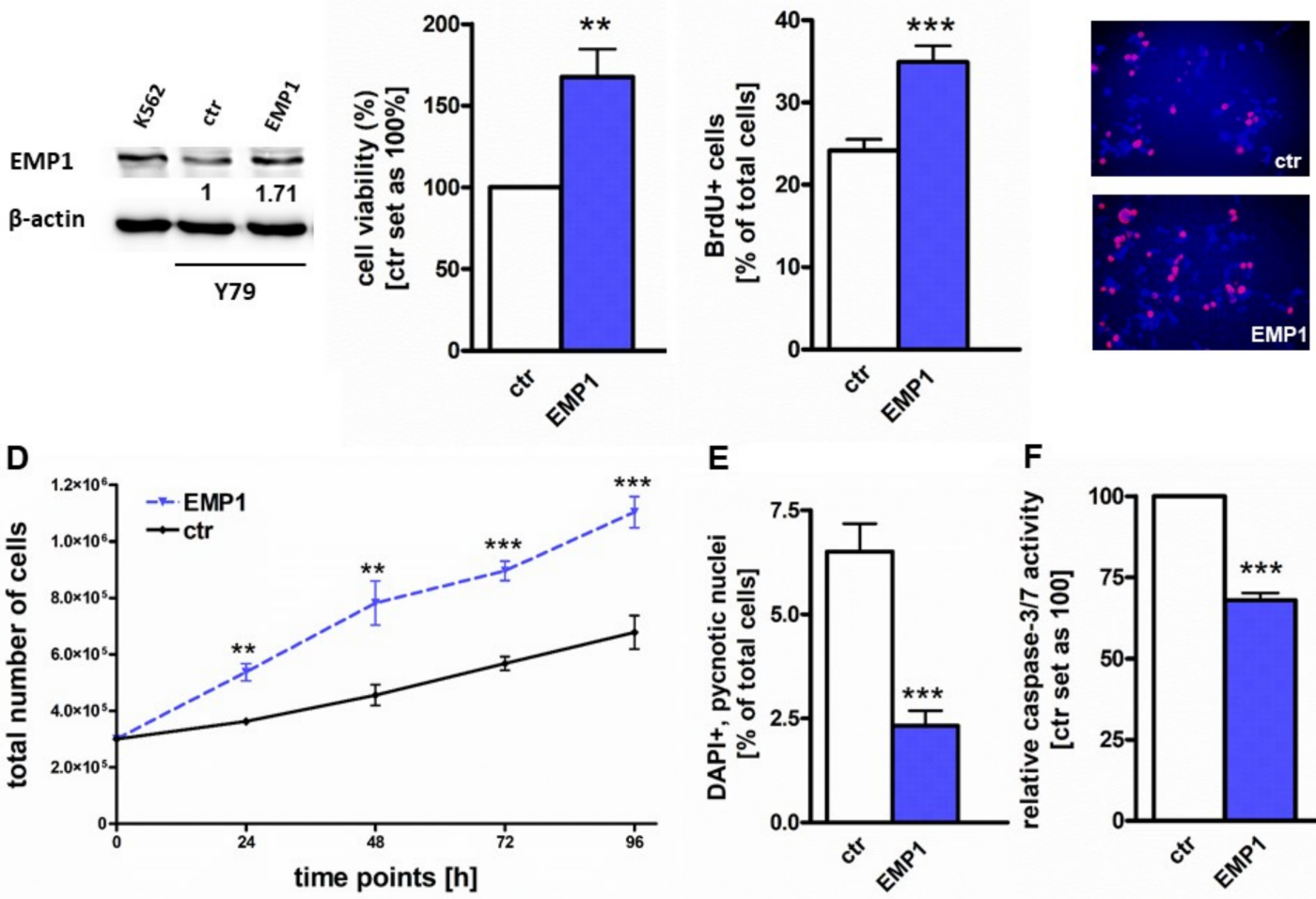

E

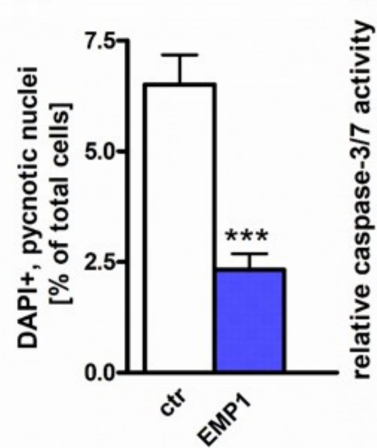

$\mathbf{F}$

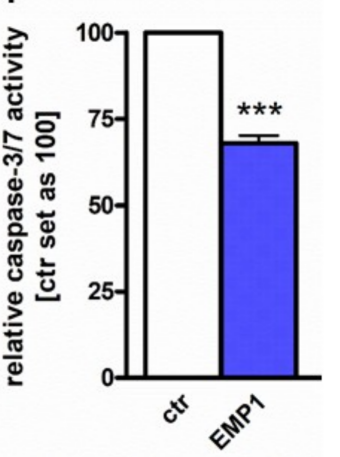

Figure 3. EMP1 overexpression leads to increased cell viability and proliferation and induces caspase-3/7 dependent apoptosis in Y79 RB cells. (A) Western blot data confirmed increased EMP1 protein levels after EMP1 overexpression (EMP1) in Y79 cells. The CML cell line K562 served as an EMP1 positive control, B-actin as a loading control. (B,C) Stably EMP1 overexpressing Y79 RB cells (EMP1) showed significantly increased viability and proliferation levels compared to control cells (ctr) as revealed by (B) WST-1 assays and (C) BrdU stains. red: BrdU-labeled cells; blue: DAPI counterstaining (D) Growth curve analysis of EMP1 overexpressing Y79 RB cells showed a significant increase in cell growth rates. (E) EMP1 overexpressing Y79 cells (EMP1) displayed a significantly reduced apoptosis rate compared to control cells (ctr) as revealed by DAPI stains. (F) Caspase-3/7 activity was significantly reduced after EMP1 overexpression in Y79 RB cells (EMP1) compared to control cells (ctr). Values are means of at least 3 independent experiments \pm SEM. ${ }^{* *} p$-value $<0.01$; ${ }^{* * *} p$-value $<0.001$ statistical differences compared to the control group calculated by Student's $t$-test.

\subsection{EMP1 Overexpression Increases Tumorigenicity of Y79 Cells In Vitro and In Vivo}

Colony formation and soft agarose assays indicated a significant higher ability of EMP1 overexpressing Y79 cells to form colonies (Figure 4A) and to grow anchorage independently (Figure 4B).

To investigate whether EMP1 influences RB cells' tumor growth, we used the chicken chorioallantoic membrane (CAM) as an in vivo model. Y79 cells stably overexpressing EMP1 and control cells were inoculated onto the CAM of 10-day old chicken embryos. Photo-documentation of the tumors developing from Y79 cells inoculated onto the CAM (Figure 5A) and quantification of tumor size, weight and volume revealed that EMP1 overexpressing RB cells develop significantly larger tumors (Figure 5B) than control cells, exhibiting higher weights (Figure 5C) and volumes (Figure 5D). There are no significant changes in the number of developing tumors between EMP1 overexpressing cells and control cells (data not shown). 
A

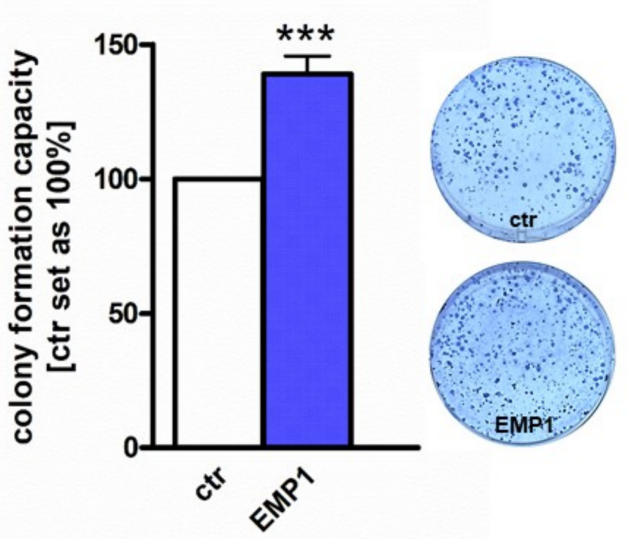

B

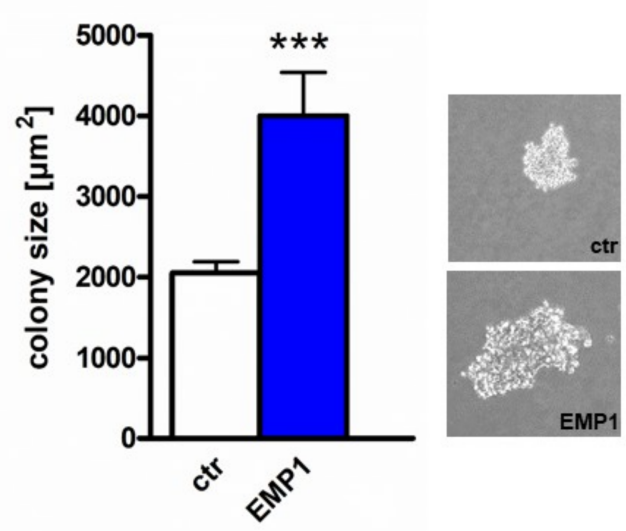

Figure 4. Effect of stable EMP1 overexpression on RB cell colony formation and anchorage independent growth. (A) Quantification of colony formation assays (CFA) showing a significant higher capacity of EMP1 overexpressing Y79 RB cells to form colonies (EMP1) compared to control cells (ctr). (B) Quantification of anchorage independent growth capacity of EMP1 overexpressing Y79 RB cells (EMP1) compared to control cells (ctr) as revealed by soft agarose assay. All photographs are taken 3 weeks after seeding EMP1 overexpressing and control Y79 RB cells. Values are means of at least 3 independent experiments \pm SEM. ${ }^{* * *} p$-value $<0.001$; statistical differences compared to the control group calculated by Student's $t$-test.

A

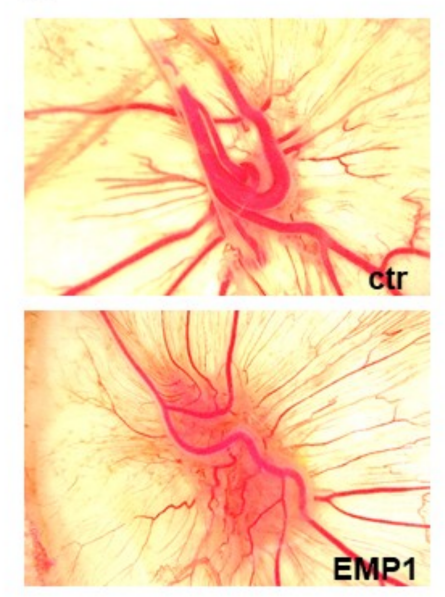

B

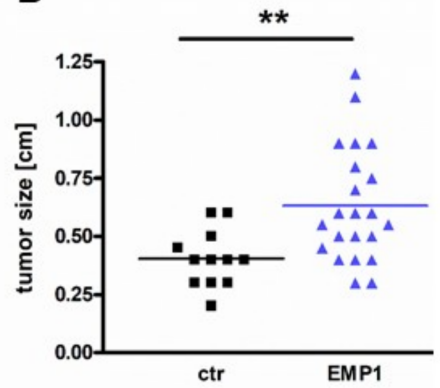

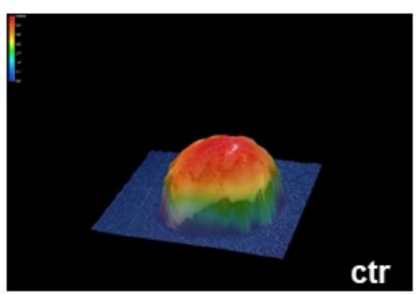

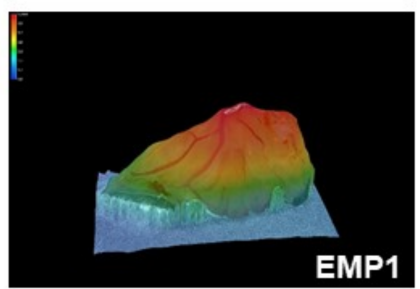

C

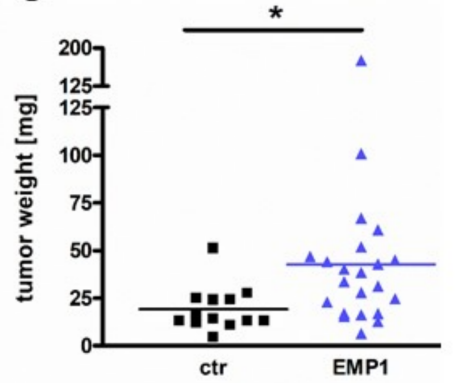

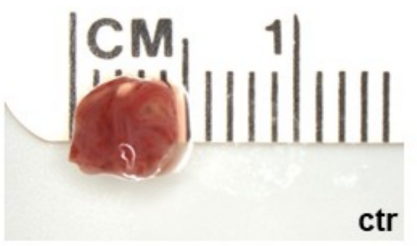

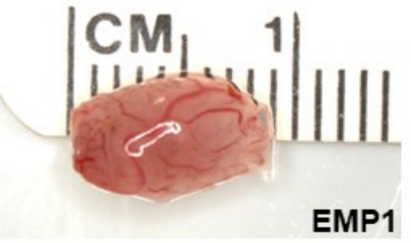

D

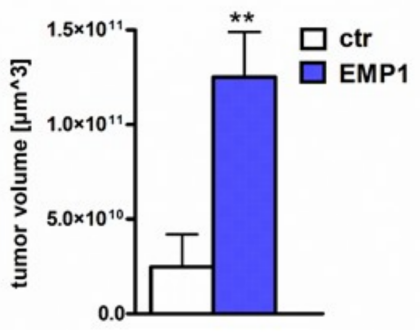

Figure 5. Stable, lentiviral EMP1 overexpression increases tumor formation capacity of Y79 RB cells. (A) Photographs of CAM tumors in situ (left column), 3D tumor volume (middle column) and ruler measurements (in $\mathrm{cm}$ ) of excised tumors (right column) revealing that tumors forming in the upper 
CAM after grafting EMP1 overexpressing Y79 RB cells were significantly larger compared to those developing from control cells (ctr). (B,C) Quantification of CAM assays by (B) tumor size, (C) tumor weight and (D) tumor volume. Values are means from at least 3 independent experiments (except for tumor volume which was measured exemplarily in one experimental setting) \pm SEM. * $p$-value $<0.05$, ** $p$-value $<0.01$ statistical differences compared to the control group calculated by Student's $t$-test.

\subsection{MiR-34a and EMP1 Overexpression Enhances Chemosensitivity of Y79 RB Cells}

As a previous study revealed that overexpression of miR-34a in RB cells leads to enhanced chemosensitivity against chemotherapeutics [23] and EMP1 has been shown to be regulated by miR-34a in RB cell lines [24], we analyzed the effect of miR-34a and EMP1 overexpression on Y79 cells' sensitivity towards the common RB chemotherapeutics etoposide, vincristine and cisplatin.

Compared to untreated controls, apoptosis levels of untreated miR34a overexpressing Y79 cells significantly increased after miR-34a overexpression. Additional treatment with low doses $(0.01 \mu \mathrm{M})$ of the chemotherapeutics vincristine, etoposide, and cisplatin significantly augmented this effect (Figure 5A) indicating that miR34a overexpression increases Y79 cells' chemosensitivity towards VEC chemotherapeutics. Cell viability assays confirmed this notion as not only the viability of miR34 overexpressing Y79 cells significantly decreased, but subsequent application of VEC chemotherapeutics yet resulted in an additional decrease of viability levels except for vincristine treatment (data not shown).

In order to test if miR-34a induced chemosensitivity is mediated via downstream regulation of EMP1, we analyzed the effect of EMP1 overexpression on Y79 cells' resistance towards commonly used RB chemotherapeutics. As shown before (Figure 3E), apoptosis levels of untreated Y79 cells significantly decreased after EMP1 overexpression (Figure 6B). In EMP1 overexpressing cells, however, additional treatment with high doses of VEC chemotherapeutics resulted in significantly enhanced apoptosis levels compared to respective control cells, except for cisplatin treatment (Figure 6B), indicating that contrary to our expectations, EMP1 overexpression does not increase the resistance of Y79 cells towards cytostatic drugs, but likewise increases chemosensitivity of RB cells.
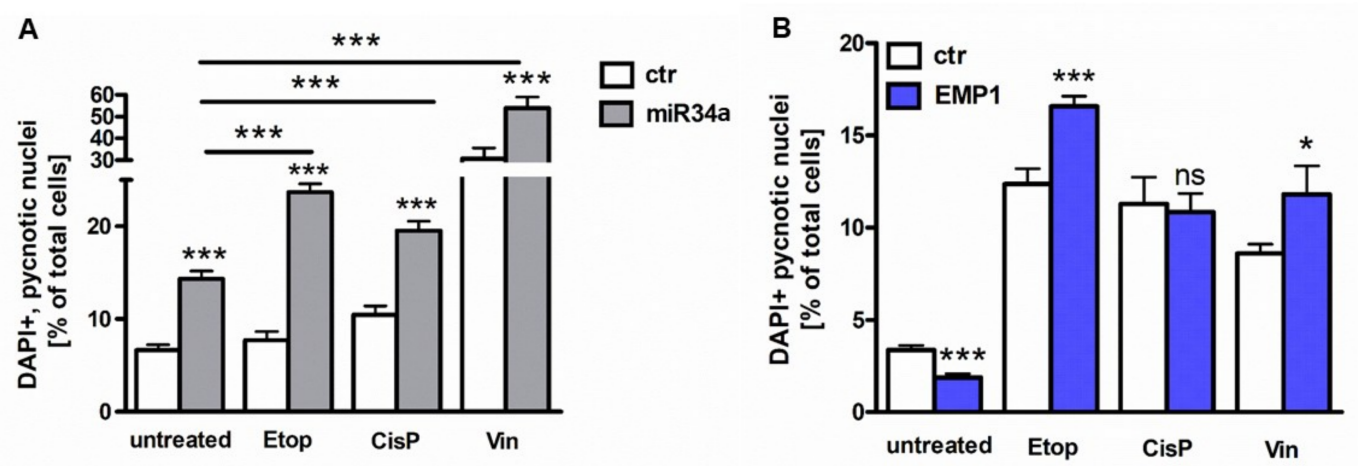

Figure 6. MiR-34a and EMP1 overexpression leads to enhanced chemosensitivity. (A) Overexpression of miR-34a in Y79 RB cells leads to significantly increased apoptosis levels compared to control cells (ctr). Additional treatment with etoposide (Etop), cisplatin (CisP), or vincristine (Vin) significantly elevates the apoptosis levels compared to untreated miR-34a overexpressing Y79 RB cells. (B) Overexpression of EMP1 in Y79 RB cells leads to significantly decreased apoptosis levels compared to control cells (ctr). Additional treatment with etoposide (Etop) and vincristine (Vin) leads to higher apoptosis levels compared to untreated EMP1 overexpressing Y79 RB cells. Additional treatment with cisplatin (CisP) did not change the apoptosis level compared to untreated EMP1 expressing Y79 RB cells. Values are means of at least 3 independent experiments \pm SEM. ${ }^{*} p$-value $<0.05,{ }^{* * *} p$-value $<0.001$; statistical differences compared to the control group calculated by one-way ANOVA and Newman-Keuls post test. 


\section{Discussion}

The role of TFFs in cancer progression of different tumor entities has been largely described in the literature [26,27]. Especially the function of TFF3 is controversially discussed as it is considered as a potential oncogenic factor or a tumor suppressor gene depending on the tissue investigated $[26,28]$. Ectopic TFF3 expression in cancer tissues like gastric, pancreatic, hepatocellular, colon, and breast cancer is linked to enhanced cell proliferation and survival as well as increased invasion and migration [13,29]. By contrast, in most thyroid tumors and in retinoblastoma TFF3 expression is decreased $[19,30]$. We demonstrated previously that this reduction in TFF3 expression in RB cell lines is regulated epigenetically [18]. In addition, we could recently show that TFF3 overexpression in four different RB cell lines has anti-proliferative and pro-apoptotic effects in vitro and leads to reduced tumor growth in vivo, supporting the notion of TFF3 as a tumor suppressor in retinoblastoma [19]. In the present study, we investigated the mechanisms underlying TFF3 mediated effects exemplarily in the RB cell line Y79. In accordance with findings of Soutto et al., who showed a TFF1 mediated p53 activation in gastric cancer cells [20], our group recently demonstrated that in RB cell lines TFF1 overexpression is likewise related to p53 activation [21]. We therefore hypothesized that p53 activation in RB cells might also be induced by TFF3 overexpression and the study presented proved this hypothesis right. We could show increased p53 protein levels and a significant activation of p53 in TFF3 overexpressing Y79 RB cells. Which binding factors are influenced by TFF3 resulting in p53 induction, needs to be revealed in future studies.

Next, we analyzed the expression levels of the known p53 microRNA target miR-34a [31], which was concordantly upregulated after TFF3 overexpression. Ectopic re-expression of miR-34a was already correlated to apoptosis and growth inhibition in multiple myeloma cells [32] and Dalgard et al. showed that miR-34a functions as a tumor suppressor in RB cells, highlighting it as a potential therapeutic target [24]. In line with these findings treatment with miR-34a mimics in lung cancers has been evaluated as a replacement therapy as miR-34a reduces lung tumor growth [22]. These effects are at least partially mediated by the direct post-transcriptional repression of postulated miR-34a target genes. Among other oncogenic substrates of miR-34a EMP1 is one of the predicted targets, regulated by miR-34a overexpression in RB cells lines [24]. The integral membrane glycoprotein EMP1 is commonly expressed in epithelial tissues of the gastrointestinal tract, skin, lungs, and brain [33,34]. Alterations of EMP1 expression have been linked to a variety of human cancers like gliomas, gastric cancer as well as in oral, laryngeal, scirrhous gastric, and esophageal cancers [35-38]. However, the function of EMP1 is controversially discussed in the literature. In most cancers, tumor suppressive functions for EMP1 have been described like inhibition of cell growth and metastasis by induction of apoptosis and prevention of angiogenesis [39]. In these tumor entities loss of EMP1 expression correlates with poor prognosis. By contrast, EMP1 silencing in T-ALL cell lines leads to increased apoptosis levels, reduced cell survival, and sensitized the cells towards prednisolone treatment [40]. In these cancers high endogenous EMP1 expression correlates with poor patients' outcome. In line with these findings, silencing EMP1 in chondrocytes inhibits cell proliferation [41].

In the present study, we detected a significant reduction in EMP1 expression levels after TFF3 overexpression in Y79 cells, accompanied by miR-34a upregulation. Thus, we set out to verify if EMP1 knockdown will lead to the same functional effects as TFF3 overexpression. We could show that knocking down EMP1 in Y79 cells indeed mimics TFF3 overexpression effects leading to reduced cell viability and proliferation and increased apoptosis levels. In order to show the tumor inductive potential of EMP1 in RB cells, we additionally overexpressed EMP1 in Y79 cells expecting to see the opposite effects following TFF3 overexpression, namely higher cell viability and proliferation levels as well as decreased cell death levels. Indeed, EMP1 overexpression in Y79 cells resulted in an induction of cell viability, concomitantly increased proliferation rates and reduced caspase3/7 dependent apoptosis levels. Additionally, colony formation capacity and anchorage independent growth were increased after EMP1 overexpression compared to control cells. The in vitro experiments were confirmed in vivo 
as EMP1 overexpressing Y79 cells displayed a higher tumorigenicity, resulting in significantly larger CAM tumors.

In line with our results, Johnson et al. showed a correlation between TFF1 expression and EMP1 regulation. Gene expression analyses of Tff1-/- mice revealed that EMP1 is significantly upregulated when Tff1 expression is lacking [34]. By contrast, our present study revealed that compared to healthy human retina tissue the endogenous EMP1 expression seems to be reduced in different RB cell lines and primary RB tumors lacking TFF3 expression (Figure S1A). This might be an effect of the relatively high endogenous miR-34a expression levels in RB cells (Figure S1B). MiR-34a is currently considered to be a promising therapeutic agent, which increases chemosensitivity and decreases aggressiveness of cancer cells and as a potential prognostic biomarker for treatment strategies. Several publications indicate a role for miR-34a in chemotherapy response in a number of tumor models including NSCLC, HCC, breast cancer, bladder cancer, colorectal cancer, prostate cancer, and head and neck squamous cell carcinoma (for review see: [31]). A higher miR-34a expression increases the sensitivity to cisplatin for lung and bladder cancer cells [42,43] as well as for RB cells to treatment with vincristine, etoposide, or carboplatin [23]. In the study presented, we confirmed an increase in chemosensitivity to the RB VEC chemotherapeutics vincristine, etoposide and cisplatin in miR-34a overexpressing Y79 cells. We also addressed the question if the chemosensitizing effects are exclusively induced by miR-34a or if the effects could also be mediated by downstream regulation of EMP1. In this respect, we overexpressed EMP1 in Y79 cells and additionally treated them with VEC chemotherapeutics in order to prove if a higher EMP1 expression leads to chemoresistance. However, in comparison to control cells no augmented chemoresistance was detectable after combined EMP1 overexpression and VEC treatment, but, contrarily, an increase in apoptosis levels was observed after treatment with etoposide and vincristine following EMP1 overexpression. The enhanced sensitivity towards cytostatic drugs observed in EMP1 overexpressing Y79 cells is most likely mediated by the pro-proliferative effects of EMP1 overexpression itself, as rapidly dividing cells are potentially more susceptible to the topoisomerase II inhibitor etoposide and vincristine-mediated tubulin binding. Thus, in RB cells the chemosensitizing effects of miR-34a are not mediated by regulation of EMP1.

In summary, we could demonstrate that the overexpression of TFF3 in Y79 RB cells leads to an activation of $\mathrm{p} 53$ and an upregulation of $m i R-34 a$, a direct target of p53. Moreover, the expression of the predicted miR34a target EMP1 was reduced after TFF3 overexpression. Taken together, our data strengthen the tumor suppressive function of TFF3 in retinoblastoma cells and emphasize TFF3's downstream signaling components miR-34a and EMP1 as potential targets for adjuvant RB therapy and drug development strategies.

\section{Material and Methods}

\subsection{Human Retina and Retinoblastoma Samples}

Post mortem human retina samples from cornea donors and retinoblastoma samples were used for comparative expression studies. The Ethics Committee of the Medical Faculty of the University of Duisburg-Essen approved the use of human retina (approval \# 06-3021, 22 March 2006) and retinoblastoma samples (approval \# 14-5836-BO, 07 May 2014) for research conducted in the course of the study presented and written informed consent has been obtained from patients' relatives or parents.

\subsection{Cell Culture}

The human retinoblastoma cell lines Y79 [44,45], originally purchased from the Leibniz Institute DSMZ (German Collection of Microorganisms and Cell Cultures) were kindly provided by Dr. H. Stephan. All RB cell lines used in this study were cultivated as described previously [45].

Human embryonic kidney cells (HEK293-T) were grown as adherent cell cultures in DMEM with $10 \%$ FBS, $4 \mathrm{mM} \mathrm{L}$-glutamine, $100 \mathrm{U}$ penicillin/mL and $100 \mu \mathrm{g}$ streptomycin $/ \mathrm{mL}$ at $37^{\circ} \mathrm{C}, 5 \% \mathrm{CO}_{2}$, and $95 \%$ humidity. 


\subsection{Treatment with Chemotherapeutics}

To investigate chemosensitivity, miR34a or EMP1 overexpressing Y79 cells and control cells transfected with an empty vector control were treated individually with three different chemotherapeutics (vincristine, etoposide, and cisplatin) and compared to untreated cells. Twenty-four hours before the transient transfection with miR-34a, $2 \times 10^{5} \mathrm{Y} 79$ cells/mL were seeded in growth medium without antibiotics. Twenty-four hours after transient transfection, Y79 cells were treated with low effective doses $(0.01 \mu \mathrm{M})$ of vincristine, etoposide or cisplatin for $72 \mathrm{~h}$, identified by previous concentration analyses. Y79 cells stably transduced with EMP1 lentiviral particles were treated with highly effective doses of $2.5 \mathrm{nM}$ vincristine, $1 \mu \mathrm{M}$ etoposide or cisplatin for $72 \mathrm{~h}$ as previously described by Liu et al. [23]. After $72 \mathrm{~h}$ of treatment with chemotherapeutics cell viability was analyzed by WST-1 assay and apoptosis via DAPI cell counts.

\subsection{Plasmids and Lentiviral Expression Vectors}

To generate a lentiviral EMP1 expression vector (pLenti CMV_EMP1), the human $E M P 1$ cDNA sequence was amplified from the RC208410 vector (Origene) using the forward primer 5'-CGAATTCTATGTTGGTATTGCTGGCTGG-3' and the reverse primer 5'-CGCTCGAGTTATTTCTTTCTCAGGACCAG-3' containing EcoRI and Xhol restriction sites (underlined). The PCR product was cloned into the pCRII-TOPO vector (Invitrogen), excised by digestion with EcoRI and XhoI and ligated into the pENTR4 vector, digested with the same restriction enzymes. EMP1 was finally cloned into the pLenti CMV Puro Dest vector (Addgene) by the use of a Gateway LR Clonase II Enzyme Mix (Invitrogen), following the manufacturer's protocol. The "Mission shRNA Plasmid DNA" with a pLKO.2-puro backbone used in the EMP1 knockdown experiments was purchased from Sigma-Aldrich (St. Louis, MI, USA), (shEMP1 cloneTRCN0000117946). The non-mammalian shRNA control pPRIME-CMV-Neo-FF3 (p234) containing a targeting hairpin sequence against firefly-luciferase was used in all transduction experiments [46].

To generate a miR34a expression vector (pSG5-miR34a), the human miR34a sequence was amplified from cDNA of HEK293T cells by RT-PCR using the forward primer 5'-CGGAATTCGCATCCTTTCTTTCCTCCCC-3' and the reverse primer 5'-CGGGATCCGGGCATCTCTCGCTTCATCT-3' containing EcoRI and BamHI restriction sites (underlined). The PCR product was cloned into the PCRII-TOPO vector (Invitrogen, Karlsruhe, Germany), excised by digestion with EcoRI and BamHI and ligated into the pSG5 vector digested with the same restriction enzymes. All vector constructs were verified by sequencing.

\subsection{TFF3 and EMP1 Overexpression and EMP1 Knockdown in Y79 Retinoblastoma Cells}

For transient TFF3 overexpression, Y79 RB cells were transfected as previously described by our group [19].

For virus production, HEK293T cells were transfected as described previously [45] with each of the following plasmid DNAs: packaging vector pczVSV-G [47], pCD NL-BH [47], and either pLenti CMV_TFF3, pLenti CMV_EMP1, shEMP1 cloneTRCN0000117946 or pPRIME-CMV-Neo-FF3 (p234).

Experimental conditions for the lentiviral transductions were the same as described previously [19]. In addition to TFF3-coding lentiviral particles EMP1-coding particles were used.

\subsection{RNA Extraction and Quantitative Real-Time PCR}

RNA isolations from RB cells were performed using the miRNeasy Kit (Qiagen, Hilden, Germany) and the miRNeasy FFPE Kit (Qiagen), respectively.

For quantitative Real-time PCR analyses cDNA was synthesized with the QuantiTect Reverse Transcription Kit (Qiagen) following the manufacturer's protocol and the following human Taqman Gene Expression Assays (Applied Biosystems, Dreieich, Germany) were used: EMP1 (Hs0060855_m1) and 18S (Hs99999901_s1). The latter was used as an endogenous control. In RT-qPCR reactions, 
conducted in duplicates, $20 \mu \mathrm{L}$ of a TaqMan Universal PCR Master Mix (Applied Biosystems) was used and the samples were run in the following program: $50^{\circ} \mathrm{C}$ for $2 \mathrm{~min}, 95^{\circ} \mathrm{C}$ for $10 \mathrm{~min}, 95^{\circ} \mathrm{C}$ for $15 \mathrm{~s}$ for 40 cycles, $60^{\circ} \mathrm{C}$ for $60 \mathrm{~s}$.

After cDNA synthesis, we used the miScript PCR Starter Kit (\# 2181193; Qiagen) for miRNA RT-qPCR analyses, following the instructions of the manufacturer. For the quantification of mature miRNAs, a designated miScript HiSpec buffer was used together with specific primers for miR-34a (5'-TGGCAGTGTCTTAGGTGGTTGT-3') and 5.8S RNA (5'-CTACGCCTGTCT GAGCGTCGCTT-3') as endogenous control. The reactions were performed in duplicates using a 7300 Real-Time PCR System (Applied Biosystems).

\subsection{Western Blotting}

After washing in PBS, cells were lysed for $60 \mathrm{~min}$ at $4{ }^{\circ} \mathrm{C}$ in RIPA buffer plus additives (see: [15]) and cleared by a $10 \mathrm{~min}$ centrifugation step at $14,000 \mathrm{rpm}$ and $4{ }^{\circ} \mathrm{C}$. Equal amounts of protein extracts were separated on a $12 \%$ SDS-PAGE and transferred onto nitrocellulose membranes. Primary antibodies used (incubated overnight at $4{ }^{\circ} \mathrm{C}$ ): EMP1 (1:1000): ab230445, Abcam; p53 (1:400): sc-71817, Santa Cruz; TFF3 (1:500): TA307376, Origene; ß-actin (1:1000): \#4967, Cell Signalling. Secondary antibodies used: HRP-conjugated goat-anti-rabbit or rabbit-anti-mouse antibody (1:10,000): P0448 and P0260; DAKO. Signals were developed by the Western Bright Chemiluminescence Reagent (Advansta).

\subsection{Cell Viability}

To determine cell viability, $3 \times 10^{5}$ cells in $500 \mu \mathrm{L}$ medium were seeded in a 24 -well plate. After $72 \mathrm{~h}$ of incubation, $100 \mu \mathrm{L}$ cell suspensions were seeded in two triplicates into a 96 well plate. After adding $10 \mu \mathrm{L}$ of a WST (water soluble tetrazolium) solution to each well, we incubated the cells at $37^{\circ} \mathrm{C}$ for a designated period. The formazan product of viable cells was quantified in a microplate reader at an absorbance of $450 \mathrm{~nm}$.

\subsection{Growth Kinetic}

For growth kinetics analyses in a in 24-well plate format, we seeded $3 \times 10^{5}$ cells in $500 \mu \mathrm{L}$ supplemented DMEM in triplicates and determined the number of vital cells by manual cells counts every $24 \mathrm{~h}$ ( 5 time points: $0 \mathrm{~h}, 24 \mathrm{~h}, 48 \mathrm{~h}, 72 \mathrm{~h}$, and $96 \mathrm{~h}$ ) after trypan blue exclusion.

\subsection{Cell Proliferation and Apoptosis Detection}

To determine cell proliferation, $4 \mathrm{~h}$ prior to PFA fixation $5 \mu \mathrm{M}$ BrdU (5-Bromo-2'-deoxyuridine; BrdU; Sigma) were added to the cells. The BrdU signal was revealed by a rat anti-BrdU primary antibody (1:1000; ab6326; Abcam, Cambridge, UK) and visualized by an Alexa Flour 594-labelled goat anti-rat secondary antibody (1:1000; Molecular Probes, Eugene, OR, USA). Changes in cell death levels were determined by manual counts of pycnotic nuclei after 4', 6-Diamidino-2-phenylindole (DAPI; Sigma) stains.

For each experiment six coverslips were stained and the percentages of proliferating or apoptotic cells were calculated as described previously by our group [45,48].

\subsection{Caspase-Glo 3/7 Assay}

To analyze caspase 3 and 7 cleavage activity after stable EMP1 transduction of Y79 cells a caspase-Glo 3/7 assay (Promega, Madison, WI USA) was used and luminescence was measured. Therefore, $9 \times 10^{5}$ EMP1 overexpressing and control cells were seeded in $300 \mu \mathrm{L}$ growth medium (24 well plate) and incubated overnight. After $24 \mathrm{~h} 80 \mu \mathrm{L}$ of each cell suspension were mixed with $80 \mu \mathrm{L}$ of caspase-3/7 reagent and seeded in a white 96 well plate for $2 \mathrm{~h}$. Following the manufacturer's instructions luminescence was measured after $2 \mathrm{~h}$ of incubation (Orion II microplate luminometer). The reactions were performed twice in triplicates. 


\subsection{Colony Formation and Soft Agarose Assay}

For colony formation assays (CFA) 4000 cells were seeded in $2 \mathrm{~mL}$ of DMEM with supplements on poly-D-lysine coated 6 well plates. After three weeks of incubation cells were fixed and stained with a $2.5 \%$ crystal violet/10\% formalin solution for $30 \mathrm{~min}$ at RT. After three washes with water and air drying the colonies were photographed for colony density measurements with ImageJ software. Soft agarose assays were performed as described in detail previously [46]. Colony formation efficiency $(\%)$ per visual field was determined by counting colonies and single cells (in triplicates) in five visual fields $(10 \times)$ per cell line.

\subsection{CAM Assay}

In order to test for changes in tumor formation and migration capacity, EMP1 overexpressing Y79 cells and control cells were grafted on the chorioallantoic membrane (CAM) mainly following Zijlstra and Palmers protocols $[49,50]$. Twenty eggs were grafted in at least three independent experiments. Seven days after grafting (E10-17) tumors which formed from the grafted cells were excised, measured and photographed as described previously [19].

\subsection{Luciferase Assay}

TFF3 dependent p53 activity was measured with the p53 responsive reporter PG13-Luc (with p53 binding site) and the non-responsive reporter MG15-Luc (with a mutant p53 binding site; Addgene [51]) after co-transfection of pCS2+_UBQ_TFF3 or an empty control vector (pCS2+_UBQ). As controls, cells were transfected with a promotor-less pGL3-basic and a pGL3-control luciferase vector containing an SV40 promotor and enhancer. All cells were additionally co-transfected with pGL4.75 (Renilla-Luc) for normalization. Cells were lysed after $48 \mathrm{~h}$ in $1 \times$ passive-lysis buffer (Promega) and the luciferase-activity was measured with the "Dual Luciferase reporter assay system" (E1910; Promega and Glomax 20/20 luminometer) as described by the manufacturer. The relative p53 luciferase-activity was determined as the quotient of firefly-luciferase and Renilla-luciferase-activity. Analyses were performed in triplicates.

\subsection{Statistical Analysis}

All assays were performed in triplicates. Statistical analyses were performed using GraphPad Prism 6. Data represent means \pm SEM of three independent experiments from independent RB cell cultures. Results were analyzed by a Student's $t$-test or one-way ANOVA and Newman-Keuls post tests and considered significantly different if $p$-value $<0.05 \mathrm{~m}\left(^{*}\right), p$-value $<0.01\left(^{* *}\right)$ or $p$-value $<0.001\left(^{* * *}\right)$. Statistics on the growth curves was performed using a free web interface http://bioinf.wehi.edu. au/software/compareCurves/, which uses the "compare growth curves" function from a statistical modeling package called statmod, available from the "R Project for Statistical Computing": http: //www.r-project.org, previously described elsewhere [52].

Supplementary Materials: Supplementary materials can be found at http:/www.mdpi.com/1422-0067/20/17/ 4129/s1.

Author Contributions: Conceptualization, N.D. and M.B.; Methodology, S.K. and M.B. and J.G.-K. and O.S.; Validation, S.K. and M.B.; Investigation, S.K. and M.B. and J.G.-K. and O.S.; Resources, K.M. and H.S.; Data curation, N.D. and M.B.; Writing_original Draft Preparation, M.B.; Writing-Review and Editing, N.D. and S.K. and M.B.; Visualization, S.K. and M.B.; Supervision, N.D. and M.B.

Funding: This research was funded by Else-Kröner-Fresenius Stiftung foundation.

Acknowledgments: The authors would like to thank H. Hanenberg for the lentiviral vectors and Jaroslav Dankert for the psG5 vector. We also would like to thank U. Gerster for excellent technical assistance and D. Gioè for valuable help with the cell counts.

Conflicts of Interest: The authors declare no conflict of interest. 


\section{References}

1. Dimaras, H.; Kimani, K.; Dimba, E.A.O.; Gronsdahl, P.; White, A.; Chan, H.S.L.; Gallie, B.L. Retinoblastoma. Lancet 2012, 379, 1436-1446. [CrossRef]

2. Dimaras, H.; Corson, T.W. Retinoblastoma, the visible CNS tumor: A review. J. Neurosci. Res. 2018, 97, $29-44$. [CrossRef] [PubMed]

3. Corson, T.W.; Gallie, B.L. One hit, two hits, three hits, more? Genomic changes in the development of retinoblastoma. Genes Chromosom. Cancer 2007, 46, 617-634. [CrossRef] [PubMed]

4. Dimaras, H.; Khetan, V.; Halliday, W.; Orlic-Milacic, M.; Prigoda, N.L.; Piovesan, B.; Marrano, P.; Corson, T.W.; Eagle, R.C.; Squire, J.A.; et al. Loss of RB1 induces non-proliferative retinoma: Increasing genomic instability correlates with progression to retinoblastoma. Hum. Mol. Genet. 2008, 17, 1363-1372. [CrossRef] [PubMed]

5. Rushlow, D.E.; Mol, B.M.; Kennett, J.Y.; Yee, S.; Pajovic, S.; Thériault, B.L.; Prigoda-Lee, N.L.; Spencer, C.; Dimaras, H.; Corson, T.W.; et al. Characterisation of retinoblastomas without RB1 mutations: Genomic, gene expression, and clinical studies. Lancet Oncol. 2013, 14, 327-334. [CrossRef]

6. Singh, U.; Malik, M.A.; Goswami, S.; Shukla, S.; Kaur, J. Epigenetic regulation of human retinoblastoma. Tumor Boil. 2016, 37, 14427-14441. [CrossRef]

7. Yousef, Y.A.; Soliman, S.E.; Astudillo, P.P.P.; Durairaj, P.; Dimaras, H.; Chan, H.S.L.; Héon, E.; Gallie, B.L.; Shaikh, F. Intra-arterial Chemotherapy for Retinoblastoma. JAMA Ophthalmol. 2016, 134, 1202. [CrossRef]

8. Munier, F.L.; Gaillard, M.-C.; Balmer, A.; Soliman, S.; Podilsky, G.; Moulin, A.P.; Beck-Popovic, M. Intravitreal chemotherapy for vitreous disease in retinoblastoma revisited: From prohibition to conditional indications. Br. J. Ophthalmol. 2012, 96, 1078-1083. [CrossRef]

9. Hoffmann, W.; Hauser, F. The P-domain or trefoil motif: A role in renewal and pathology of mucous epithelia? Trends Biochem. Sci. 1993, 18, 239-243. [CrossRef]

10. Hoffmann, W. Trefoil factors TFF (trefoil factor family) peptide-triggered signals promoting mucosal restitution. Cell. Mol. Life Sci. CMLS 2005, 62, 2932-2938. [CrossRef]

11. Thim, L.; May, F.E.B. Structure of mammalian trefoil factors and functional insights. Cell. Mol. Life Sci. CMLS 2005, 62, 2956-2973. [CrossRef] [PubMed]

12. Kjellev, S. The trefoil factor family-Small peptides with multiple functionalities. Cell. Mol. Life Sci. CMLS 2009, 66, 1350-1369. [CrossRef] [PubMed]

13. Busch, M.; Dünker, N. Trefoil factor family peptides-Friends or foes? Biomol. Concepts 2015, 6, 343-359. [CrossRef] [PubMed]

14. McFall, R.C.; Sery, T.W.; Makadon, M. Characterization of a new continuous cell line derived from a human retinoblastoma. Cancer Res. 1977, 37, 1003-1010. [PubMed]

15. Weise, A.; Dünker, N. High trefoil factor 1 (TFF1) expression in human retinoblastoma cells correlates with low growth kinetics, increased cyclin-dependent kinase (CDK) inhibitor levels and a selective down-regulation of CDK6. Histochem. Cell Biol. 2013, 139, 323-338. [CrossRef] [PubMed]

16. Khoury, T.; Chadha, K.; Javle, M.; Donohue, K.; LeVea, C.; Iyer, R.; Okada, H.; Nagase, H.; Tan, D. Expression of Intestinal Trefoil Factor (TFF-3) in Hepatocellular Carcinoma. Int. J. Pancreatol. 2005, 35, 171-178. [CrossRef]

17. Yamachika, T.; Werther, J.L.; Bodian, C.; Babyatsky, M.; Tatematsu, M.; Yamamura, Y.; Chen, A.; Itzkowitz, S. Intestinal trefoil factor: A marker of poor prognosis in gastric carcinoma. Clin. Cancer Res. 2002, 8, 1092-1099. [PubMed]

18. Philippeit, C.; Busch, M.; Dünker, N. Epigenetic Control of Trefoil Factor Family (TFF) Peptide Expression in Human Retinoblastoma Cell Lines. Cell. Physiol. Biochem. 2014, 34, 1001-1014. [CrossRef] [PubMed]

19. Große-Kreul, J.; Busch, M.; Winter, C.; Pikos, S.; Stephan, H.; Dünker, N. Forced Trefoil Factor Family Peptide 3 (TFF3) Expression Reduces Growth, Viability, and Tumorigenicity of Human Retinoblastoma Cell Lines. PLoS ONE 2016, 11, e0163025. [CrossRef] [PubMed]

20. Soutto, M.; Chen, Z.; Saleh, M.A.; Katsha, A.; Zhu, S.; Zaika, A.; Belkhiri, A.; El-Rifai, W. TFF1 activates p53 through down-regulation of miR-504 in gastric cancer. Oncotarget 2014, 5, 5663-5673. [CrossRef] [PubMed]

21. Busch, M.; Große-Kreul, J.; Wirtz, J.J.; Beier, M.; Stephan, H.; Royer-Pokora, B.; Metz, K.; Dünker, N.; Große-Kreul, J.; Royer-Pokora, B. Reduction of the tumorigenic potential of human retinoblastoma cell lines by TFF1 overexpression involves p53/caspase signaling and miR-18a regulation. Int. J. Cancer 2017, 141, 549-560. [CrossRef] [PubMed] 
22. Yamakuchi, M.; Lowenstein, C.J. MiR-34, SIRT1, and p53: The feedback loop. Cell Cycle 2009, 8, 712-715. [CrossRef] [PubMed]

23. Liu, K.; Huang, J.; Xie, M.; Yu, Y.; Zhu, S.; Kang, R.; Cao, L.; Tang, D.; Duan, X. MIR34A regulates autophagy and apoptosis by targeting HMGB1 in the retinoblastoma cell. Autophagy 2014, 10, 442-452. [CrossRef] [PubMed]

24. Dalgard, C.L.; Gonzalez, M.; Deniro, J.E.; O’Brien, J.M. Differential MicroRNA-34a Expression and Tumor Suppressor Function in Retinoblastoma Cells. Investig. Ophthalmol. Vis. Sci. 2009, 50, 4542-4551. [CrossRef] [PubMed]

25. Wang, Y.-W.; Cheng, H.-L.; Ding, Y.-R.; Chou, L.-H.; Chow, N.-H. EMP1, EMP 2, and EMP3 as novel therapeutic targets in human cancer. Biochim. Biophys. Acta BBA Rev. Cancer 2017, 1868, 199-211. [CrossRef] [PubMed]

26. Perry, J.K.; Kannan, N.; Grandison, P.M.; Mitchell, M.D.; Lobie, P.E. Are trefoil factors oncogenic? Trends Endocrinol. Metab. TEM 2008, 19, 74-81. [CrossRef] [PubMed]

27. Emami, S.; Rodrigues, S.; Rodrigue, C.M.; Le Floch, N.; Rivat, C.; Attoub, S.; Bruyneel, E.; Gespach, C. Trefoil factor family (TFF) peptides and cancer progression. Mol. Med. TFF Pept. 2004, 25, 885-898.

28. Levak, M.T.; Mihalj, M.; Koprivčić, I.; Lovrić, I.; Novak, S.; Bijelić, N.; Lončar, M.B.; Belovari, T.; Kralik, K.; Pauzar, B. Differential expression of tff genes and proteins in breast tumors. Acta Clin. Croat. 2018, 57, 264-277.

29. Buache, E.; Etique, N.; Alpy, F.; Stoll, I.; Muckensturm, M.; Reina-San-Martin, B.; Chenard, M.P.; Tomasetto, C.; Rio, M.C. Deficiency in trefoil factor 1 (TFF1) increases tumorigenicity of human breast cancer cells and mammary tumor development in TFF1-knockout mice. Oncogene 2011, 30, 3261-3273. [CrossRef]

30. Abols, A.; Ducena, K.; Andrejeva, D.; Sadovska, L.; Zandberga, E.; Vilmanis, J.; Narbuts, Z.; Tars, J.; Eglitis, J.; Pirags, V.; et al. Trefoil factor 3 is required for differentiation of thyroid follicular cells and acts as a context-dependent tumor suppressor. Neoplasma 2015, 62, 914-924. [CrossRef]

31. Misso, G.; Di Martino, M.T.; De Rosa, G.; Farooqi, A.A.; Lombardi, A.; Campani, V.; Zarone, M.R.; Gullà, A.; Tagliaferri, P.; Tassone, P.; et al. Mir-34: A new weapon against cancer? Molecular therapy. Nucleic Acids 2014, 3, e194. [PubMed]

32. Dimopoulos, K.; Gimsing, P.; Grønbaek, K.; Grønbæk, K. Aberrant microRNA expression in multiple myeloma. Eur. J. Haematol. 2013, 91, 95-105. [CrossRef] [PubMed]

33. Yu, X.M.; Li, C.W.; Li, Y.Y.; Li, T.Y.; Pan, X.L.; Liu, J.; Bin Lin, Z.; Zhao, L.; Shi, L.; Wang, D.Y. Down-regulation of EMP1 is associated with epithelial hyperplasia and metaplasia in nasal polyps. Histopathology 2013, 63, 686-695. [CrossRef] [PubMed]

34. Taylor, V.; Welcher, A.A.; Amgen, E.P.; Suter, U. Epithelial Membrane Protein-1, Peripheral Myelin Protein 22, and Lens Membrane Protein 20 Define a Novel Gene Family. J. Boil. Chem. 1995, 270, 28824-28833. [CrossRef] [PubMed]

35. Bredel, M. Functional Network Analysis Reveals Extended Gliomagenesis Pathway Maps and Three Novel MYC-Interacting Genes in Human Gliomas. Cancer Res. 2005, 65, 8679-8689. [CrossRef]

36. Johnson, A.H.; Frierson, H.F.; Zaika, A.; Powell, S.M.; Roche, J.; Crowe, S.; Moskaluk, C.A.; El-Rifai, W. Expression of Tight-Junction Protein Claudin-7 Is an Early Event in Gastric Tumorigenesis. Am. J. Pathol. 2005, 167, 577-584. [CrossRef]

37. Wang, H.-T.; Kong, J.-P.; Ding, F.; Wang, X.-Q.; Wang, M.-R.; Liu, L.-X.; Wu, M.; Liu, Z.-H. Analysis of gene expression profile induced by EMP-1 in esophageal cancer cells using cDNA Microarray. World J. Gastroenterol. 2003, 9, 392-398. [CrossRef]

38. Kornberg, L.J.; Villaret, D.; Popp, M.; Lui, L.; McLaren, R.; Brown, H.; Cohen, D.; Yun, J.; McFadden, M. Gene Expression Profiling in Squamous Cell Carcinoma of the Oral Cavity Shows Abnormalities in Several Signaling Pathways. Laryngoscope 2005, 115, 690-698. [CrossRef]

39. Sun, G.G.; Lu, Y.F.; Fu, Z.Z.; Cheng, Y.J.; Hu, W.N. EMP1 inhibits nasopharyngeal cancer cell growth and metastasis through induction apoptosis and angiogenesis. Tumour Biol. 2014, 35, 3185-3193. [CrossRef]

40. Ariës, I.M.; Jerchel, I.S.; Dungen, R.E.S.R.V.D.; Berk, L.C.J.V.D.; Boer, J.M.; Horstmann, M.A.; Escherich, G.; Pieters, R.; Boer, M.L.D. EMP1, a novel poor prognostic factor in pediatric leukemia regulates prednisolone resistance, cell proliferation, migration and adhesion. Leukemia 2014, 28, 1828-1837. [CrossRef] 
41. Li, Z.-Y.; Xiong, S.-H.; Hu, M.; Zhang, C.-S. Knockdown Epithelial Membrane Protein 1 Suppresses Human Degenerative Intervertebral Disc-Derived Nucleus Pulposus Cell Proliferation. Cartilage 2011, 2, 300-306. [CrossRef]

42. Wang, X.; Dong, K.; Gao, P.; Long, M.; Lin, F.; Weng, Y.; Ouyang, Y.; Ren, J.; Zhang, H. microRNA-34a Sensitizes Lung Cancer Cell Lines to DDP Treatment Independent of p53 Status. Cancer Biother. Radiopharm. 2013, 28, 45-50. [CrossRef]

43. Vinall, R.L.; Ripoll, A.Z.; Wang, S.; Pan, C.-X.; deVere White, R.W. MiR-34a chemosensitizes bladder cancer cells to cisplatin treatment regardless of p53-Rb pathway status. Int. J. Cancer 2012, 130, 2526-2538. [CrossRef]

44. Reid, T.W.; Rabson, A.S.; Tralka, T.S.; Wilcox, J.L.; Albert, D.M.; Russell, P.; Craft, J.; Chu, E.W. Characteristics of an Established Cell Line of Retinoblastoma. J. Natl. Cancer Inst. 1974, 53, 347-360. [CrossRef]

45. Busch, M.; Philippeit, C.; Weise, A.; Dünker, N. Re-characterization of established human retinoblastoma cell lines. Histochem. Cell Biol. 2015, 143, 325-338. [CrossRef]

46. Stegmeier, F.; Hu, G.; Rickles, R.J.; Hannon, G.J.; Elledge, S.J. A lentiviral microRNA-based system for single-copy polymerase II-regulated RNA interference in mammalian cells. Proc. Natl. Acad. Sci. USA 2005, 102, 13212-13217. [CrossRef]

47. Hartmann, L.; Neveling, K.; Borkens, S.; Schneider, H.; Freund, M.; Grassman, E.; Theiss, S.; Wawer, A.; Burdach, S.; Auerbach, A.D.; et al. Correct mRNA Processing at a Mutant TT Splice Donor in FANCC Ameliorates the Clinical Phenotype in Patients and Is Enhanced by Delivery of Suppressor U1 snRNAs. Am. J. Hum. Genet. 2010, 87, 480-493. [CrossRef]

48. Haubold, M. Bone morphogenetic protein 4 (BMP4) signaling in retinoblastoma cells. Int. J. Boil. Sci. 2010, 6, 700-715. [CrossRef]

49. Zijlstra, A.; Mellor, R.; Panzarella, G.; Aimes, R.T.; Hooper, J.D.; Marchenko, N.D.; Quigley, J.P. A quantitative analysis of rate-limiting steps in the metastatic cascade using human-specific real-time polymerase chain reaction. Cancer Res. 2002, 62, 7083-7092.

50. Palmer, T.D.; Lewis, J.; Zijlstra, A. Quantitative Analysis of Cancer Metastasis using an Avian Embryo Model. J. Vis. Exp. 2011. [CrossRef]

51. El-Deiry, W. WAF1, a potential mediator of p53 tumor suppression. Cell 1993, 75, 817-825. [CrossRef]

52. Elso, C.M.; Roberts, L.J.; Smyth, G.K.; Thomson, R.J.; Baldwin, T.M.; Foote, S.J.; Handman, E. Leishmaniasis host response loci (lmr1-3) modify disease severity through a Th1/Th2-independent pathway. Genes Immun. 2004, 5, 93-100. [CrossRef] 